\title{
Öko-Audit am Scheideweg
}

\author{
Zum 31. Dezember 1997 sind in Deutschland 1.035 Unternehmen gemäß der \\ EG-Öko-Audit-Verordnung zertifiziert. 50 Prozent davon sind kleinere und \\ mittlere Unternehmen. Wie können deren Probleme bei der laufenden Revision \\ der Verordnung berücksichtigt werden?
}

\section{Von Heinz Kottmann}

ies war eines der Themen der Fachtagung, mit der am 13. Januar beim DIHT in Bonn das Euro-Management-Umwelt-Programm der EU seinen Abschluß fand. In dem seit Oktober 1996 laufenden größten Europäischen Öko-Audit-Projekt sind 547 Unternehmen von 51 europäischen Beratern aus 16 Ländern betreut worden. Allein in Deutschland haben die sechs ausgewählten Institutionen, unter anderem das IÖW, 74 kleine und mittlere Unternehmen (KMU) bis zur Konzeption des Umweltmanagementsystems gefuihrt. Von diesen streben 48 Unternehmen die Validierung nach der EG-Öko-Audit-Verordnung an bzw. haben die Registrierung schon vollzogen. Die etwa 70 Workshopteilnehmer befaßten sich mit den dabei gewonnenen Erfahrungen der Berater und auch einiger Unternehmen. Für die EU unterstrich Herr König von der Generaldirektion 23 „Unternehmenspolitik“ die positiven Erfahrungen in Deutschland. Aber auch in EU-Ländern, die mit der Umsetzung noch weit hinterherhinken, wie Italien oder Griechenland, war der Zuspruch des Projektes außerordentlich groß. Der deutsche Projektkoordinator Adolf von Röpenack wies auf die besondere Bedeutung der Kammern und Verbände für eine weitergehende Unterstützung der Unternehmen hin. Vertreter des Zentralverbands des Handwerks $(\mathrm{ZdH})$ und des Deutschen Industrie und Handelstages (DIHT) äußerten in vielen Punkten scharfe Kritik, stellten sich aber gleichzeitig im Grundsatz hinter die freiwillige Verordnung.

Deutlich wurde die klare Fokussierung auf eine kostengünstige Beratung. Hendric Hallay von der Unternehmensberatung ecco bezeichnete 10.000 Mark als Obergrenze der Öko-AuditKosten für Kleinunternehmen. Mehrere Berater, wie auch das IÖW, realisierten eine entsprechende effiziente Beratung durch branchenorientierte Arbeitsgruppen, in denen die Inhalte vermittelt und Arbeitsaufgaben bis zur nächsten Sitzung erteilt wurden.
Ein allgemeiner Tenor war die Beibehaltung der hohen Anforderungen der Verordnung bei der nun laufenden Revision. Hierzu nahm Klaus Krisor von der zuständigen Generaldirektion 11 „Umwelt“ der EG-Kommission erstmals Stellung. Im Anhang soll die überarbeitete Verordnung, mit deren Inkrafttreten im Jahr $2000 \mathrm{zu}$ rechnen ist, fast komplett die ISO-Norm 14.001 übernehmen, um die Praktikabilität und Verständlichkeit sowie die Kompatibilität zu den ISO-Normen zu gewährleisten. Hierbei soll allerdings der Unterschied zwischen dem „Rolls Royce“ EG-ÖkoAudit-Verordnung und dem „Lada“ ISO-Norm gewahrt bleiben. Walter Hirche, Staatssekretär im Umweltministerium, verdeutlichte die deutschen Positionen im Rahmen der laufenden Revisionsverhandlungen. Das BMU kann sich eine Ausweitung auf die Produktwerbung vorstellen, ohne die Verbraucher aufgrund der bewährten Produktkennzeichnung verwirren zu wollen. Wesentlich ist dem BMU eine Beibehaltung des Standortprinzips. Es verbindet hiermit aber auch Deregulierungspotentiale. Adolf von Röpenack wies darauf hin, daß von den Projektunternehmen keines die Deregulierung von sich aus angeführt hat, daß dieses aber allgemein als Vertrauensvorschuß und positives Signal gewertet würde. Heinz Kottmann problematisierte für das IÖW den mit einer Abschwächung verbundenen Glaubwürdigkeitsverlust und wies auf das Bedürfnis der Unternehmen nach klar verständlichen Vorgaben einhergehend mit einer Entbürokratisierung hin.

Die Tagungsunterlagen und Erfahrungsberichte zum Euromanagement sind in der IÖW-Schriftenreihe 125/97 "ÖkoAudit für kleine und mittlere Unternehmen" veröffentlicht.

\begin{tabular}{l}
\multicolumn{1}{c}{ Der Autor } \\
Heinz Kottmann ist wissenschaftlicher \\
Geschäftsführer des IÖW. \\
Kontakt: IÖW, Giesebrechtstr. 13,10629 Berlin, \\
Tel. 030 / 88459 40, Fax 8825439.
\end{tabular}

\section{Hauptsache: Ökologie}

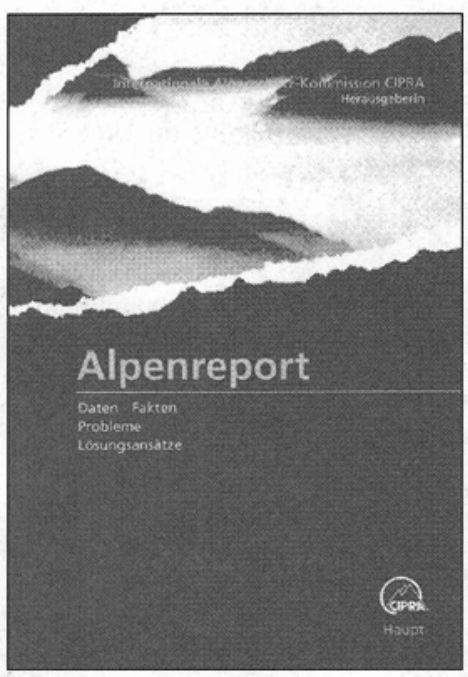

Internationale Alpenschutz-Kommission CIPRA (Herausgeberin)

Alpenreport

Daten, Fakten, Probleme, Lösungsansätze

1998. 470 Seiten, viele Abbildungen und Grafiken. DM 43.- / öS 314.- / Fr. 38.-

M. Prehn / B. Schwedt / U. Steger Verkehrsvermeidung - aber wie? Eine Analyse theoretischer Ansätze und praktischer Ausgestaltungen auf dem

Weg zu einer wirtschafts-

und umweltverträglicheren

Verkehrsentwicklung

1997. "Umwelt und Verkehr». Band 1.

212 Seiten, 5 Abbildungen, 13 Tabellen, DM 42.- / ÖS 307.- / Fr. 37.50

\section{Riedl / B. Schwedt / U. Steger /} P. Tiebler

Mobilität statt Ökologie?

Workshopberichte über konsensfähige Wege zur Lösung des Dilemmas 1998. «Umwelt und Verkehr». Band 2. 217 Seiten, 7 Abbildungen, 2 Tabellen, DM 42.- / ÖS 307.- /Fr. 37.50

Oddkjell Volle

\section{Die ökologische Herausforderung} der Getränkekartonindustrie

1997. «Wirtschaft und Ökologie». Band 11. 255 Seiten, 26 Abbildungen, 2 Tabellen, 13 Fallbeispiele, DM 76.-/öS 555.-/Fr. 68.-

Verlag Paul Haupt Bern - Stuttgart - Wien Falkenplatz $14 \cdot \mathrm{CH}-3001$ Bern

Tel. $0041 \cdot 31 \cdot 3012345 \cdot$ Fax 0041 $31 \cdot 3014669$ E-Mail: verlag@haupt.ch·www.haupt.ch 
(c) 20I0 Authors; licensee IÖW and oekom verlag. This is an article distributed under the terms of the Creative Commons Attribution Non-Commercial No Derivates License (http://creativecommons.org/licenses/by-nc-nd/3.o/), which permits unrestricted use, distribution, and reproduction in any medium, provided the original work is properly cited. 